MORRIS, Jan

Trieste o el sentido de ninguna parte

Traducción de Lucía BARAHONA.

Madrid: Gallo Nero Ediciones, 2017, 220 p.

Covacich, Mauro

La città interiore

Milano: La nave di Teseo, 2017, 233 p.

\title{
L'effetto Trieste
}

Per presentare la giornalista gallese Jan Morris, niente di meglio delle parole che le dedica il suo collega triestino ne La città interiore: "L'autrice, molto nota nel mondo anglosassone per i suoi travelogue [è anche corrispondente del Times], arriva a Trieste [nel 1945] con il primo contingente inglese nei panni [del capitano] James Morris, uomo. Scrive a lungo nella versione maschile. Firma i suoi libri col nome di James fino al 1972» quando assume l'identità femminile di Jan, con tutto ciò che comporta visto che, tra l'altro, era anche sposato-a e 'padre' di cinque figli. Covacich assume il suo caso come paradigma di una città incline a cambiare i connotati: «Non è facile dire quanto possa aver influito Trieste nelle sue scelte, ma certo la scrittrice coglie proprio nell'indeterminatezza la caratteristica principale della città: nell'indeterminatezza, ovvero nell'oscillazione di un'identità volta a volta iperborea e mediterranea, ma anche asburgica, italiana, slava, greca e profondamente ebraica, o forse proprio nella fluttuante simultaneità di ognuna di esse». Nel suo Trieste and the meaning of nowhere, pubblicato in Spagna dalle edizioni Gallo Nero (in Italia è apparso nel 2014 per i tipi de il Saggiatore con il titolo di Trieste o del nessun luogo), l'autrice mostra in effetti di avere con la città un feeling assai particolare: «Hay momentos en mi vida en los que Trieste se presenta con tal exactitud en mi inconsciente que, dondequiera que esté, me siento transportada hasta allí. Es una sensación comparable a esos instantes arcanos de quietud que en ocasiones interrumpen una conversación absolutamente banal, en los que se dice que ha pasado un ángel y en esos momentos de súbita quietud no vuelvo simplemente a visitar el lugar, sino que me reexamino también a mí misma». In quei momenti dice di avvertire una «añoranza indeterminada (lo que los galeses llaman hiraeth)» («un languore non meglio precisato -si legge nella versione italiana- che si esprime in un sentimento dolce e amaro e in uno struggimento per qualcosa che non sapremmo definire»): è il cosiddetto 
«efecto Trieste», "como si durante un breve instante proverbial me hubiera escapado del tiempo a ninguna parte». Lo statuto anomalo della città viene definito con formule assai suggestive: «alegoría [laica] del limbo» o «luogo in cui si può andare alla deriva pensando ad altro». "Persino la stessa collocazione geografica [cito ancora dall'edizione italiana] contribuisce a destare questa impressione. Pare che si trovi sempre su una piega della carta geografica, inaccessibile e remota». Il libro di Morris non è classificabile come libro di viaggio o di storia, né come memoria o elegia, ma è tutte queste cose insieme. In effetti anche gli appuntamenti di Trieste con la storia vengono evocati in un clima di evanescente suggestione: ad esempio il corteo funebre dell'arciduca Francesco Ferdinando e della moglie Sofia, assassinati a Sarajevo, le cui bare sbarcarono a Trieste dalla corazzata Viribus Unitis prima di intraprendere il viaggio in treno verso Vienna. Fu in quella circostanza, secondo Morris, in cui la città cominciò ad essere giudicata e forse anche a sentirsi, triste, anche se «mucho antes la melancolía había encontrado en la ciudad su debida imagen» nel castello di Miramare, voluto da Massimiliano d'Asburgo, «un luogo in cui il nume tutelare è il rimpianto». All'origine della malinconia vi è pure, secondo la Morris, l'altopiano del Carso, una sorta di cicatrice nel paesaggio cittadino: «Su presencia forma parte de la conciencia de la ciudad, quizá es el origen de su melancolía, puesto que antes de los ilirios, de los romanos, de los austriacos o de los italianos, el árido y rocoso karst siempre estuvo allí. Mar taciturno a un lado, amenazador karst al otro». L'altopiano del Carso incombe, non solo su Trieste, ma sull'intera penisola, con il ricordo degli innumerevoli caduti della prima mondiale, a cui vanno aggiunte le vittime trucidate dai partigiani di Tito e gettate nella vicina foiba di Basovizza, dichiarata nel 1992 monumento nazionale. Furono degli archeologi a scoprire negli anni settanta «en mitad de la fosa [...] una masa enredada de cadáveres humanos, hombres y mujeres [...] algunos de los cuales fueron encadenados todavía en vida a los muertos».

Un'altra data emblematica nella storia della città è il 1954, quando il Governo Militare Alleato cedette l'amministrazione civile all'Italia e «quel porto di mare affranto e confuso ricevette la sua sistemazione e Trieste diventò quello che è sempre rimasta da allora: un'anomalia geografica e storica, italiana per sovranità ma più o meno sola per temperamento». Ci sono, tra le altre cose, i cognomi ad illustrare l'anomala condizione della città, «crisol de razas y de clases»: cognomi che, come ricorda anche Claudio Magris in Microcosmi e come testimonia, in carne ed ossa, Mauro Covacich ne La città interiore, hanno subito a seconda delle circostanze politiche, diversi 'aggiornamenti'. Cosí, nel suo primo soggiorno a Trieste, davanti alla «enigmática variedad» dei nomi che figurano nel monumento ai caduti situato nel colle di San Giusto, Morris si «era arrovellato su quella lapide che dichiarava di onorare i morti della prima guerra mondiale senza spiegare per quale paese avessero sacrificato la vita».

Tra gli illustri visitatori della città, si citano François-René de Chateubriand che la definì «el último suspiro de la civilización» minacciata dalla «barbarie» dei Balcani; Henri Beyle (Stendhal) che fu console francese nel 1830; Sigmund Freud che vi soggiornò nel 1876, con una borsa di studio concessagli per stu- 
diare il sesso delle anguille ma senza risultati di rilievo, per cui «regresó a Viena con las manos vacías, por así decirlo, aunque quizá inspirado para elucubrar de un modo más exacto sobre el complejo de castración»; Rainer Maria Rilke al quale "cierto día de 1912, la bora inspiró las elegías modernas más queridas por los lectores». L'ammiratore straniero più entusiasta fu comunque James Joyce: «tengo la impresión de que Joyce y Trieste fueron hechos el uno para el otro». Dello scrittore irlandese si ricorda anche l'effetto benefico da lui avuto sulla letteratura italiana del Novecento: «al leer un manuscrito del tímido y joven empresario que firmaba con el seudónimo Italo Svevo, reconoció de inmediato el trabajo de un artista que, como él mismo, vivía en un destierro metafórico, rodeado de oficinistas y contables».

«Así pues —afferma la scrittrice nell'epilogo— gran parte de este pequeño libro ha consistido en una descripción de mí misma. Escribo sobre exiliados de Trieste, pero por lo general también yo misma me he sentido exiliada. Durante ańos me sentí exiliada de la normalidad, y ahora siento que soy una de esas personas exiliadas en el tiempo. El pasado es un país extranjero, pero también lo es la vejez, y una vez ingresas en ella sientes como si te encontraras en un terreno desconocido, dejando incluso tu propia tierra atrás».

Tanto per rimanere in tema di esilio, La città interiore, di Mauro Covacich (finalista al Premio Campiello del 2017) debutta con un esergo del sudafricano William Kentridge che lascia il lettore senza fiato: «La nostalgia è non avere patria nel tempo». La frase introduce i frequenti interrogativi che l'autore semina qua e là con apparente nonchalance («Se sono italiano, perché mi chiamo Covacich?»; oppure, quando di fronte alla 'vulgata' triestina che gli viene propinata sin da bambino, secondo la quale «Taliani sono i meridionali, ma anche i veneti o i milanesi, in fondo taliani sono tutti coloro che stanno a ovest di Monfalcone», il piccolo Covacich si chiede giustamente: «Ma se taliani xe lori, noi chi semo?»), tali affermazioni suggeriscono appunto una dimensione di 'non appartenenza' in cui vivono gli «italiani sbagliati», come si era auto-definito lo scrittore di origine istriana Pier Antonio Quarantotti Gambini. In un libro precedente, Trieste sottosopra (Laterza, 2006) Covacich aveva cercato di offrire una versione 'alla buona' dell'identità triestina: «Per essere più chiaro, i triestini ai mondiali fanno il tifo per gli azzurri e si commuovono quando sentono la fanfara dei bersaglieri, ma sanno, magari in modo inconscio, che la loro storia è molto più complessa di quello che dice la loro carta d'identità. Sanno che basta andare indietro di tre-quattro generazioni per scoprirsi greci, austriaci, sloveni, croati, ungheresi. Sanno che questa città è stata teresiana, napoleonica, austroungarica, fascista, per un attimo anche titina, e poi, per un bel po', americana. Sanno che l'elenco telefonico è tutto pieno di Bevilacqua che si chiamavano Vodopivec [vedi l'epopea degli «slittamenti onomastici» già citata da Jan Morris e Claudio Magris] , di Giannelli che si chiamavano Janesich [...] e che essere italiani a Trieste significa anche non dimenticare tutto questo". Questo di Covacich, lo si può definire un vertiginoso romanzo di formazione (autobiografica) a ritroso, che attraversa varie generazioni della sua famiglia con il loro relativo contesto storico, diverse frontiere geografiche (Istria, 
Bosnia, Croazia, Slovenia, ecc.) e naturalmente la letteratura (Svevo, Slataper, Quarantotti Gambini, Saba, Tomizza, Magris, Pahor, ecc.). E attraversa, anzi per meglio dire, sconfina nel territorio della finzione, costruendo sequenze 'entrañables': il nonno "comunista autodidatta» interrogato nel quartier generale degli alleati proprio dal capitano James Morris, mentre fuori attendono incuriositi "gli amici jugoslavi corsi a occupare la città per liberarla meglio"; il primo incontro, in una balera, fra i genitori, giovanissimi, con lui che per fare colpo su quella bella istriana esibisce un pacchetto di Astor, le «sigarette della domenica»; inoltre James Joyce in un sordido postribolo triestino ( $D a i$ Giacomin, fame veder de cos'che te son bon»), ecc. Naturalmente è la memoria, autentica e tenace, ad avere la meglio come nella bellissima inquadratura di nonna Lisa, venuta dal Sud, che dà una mano all'economia familiare facendo la parrucchiera, affacciata alla finestra con la sigaretta fra le dita, dopo una giornata di lavoro trascorsa fra tinture e permanenti, mentre scruta dall'alto quello strano mondo dove imperversa la bora: «Lei alla finestra, che guarda allontanarsi l'ultima cliente della giornata, le ciocche ancora turgide di lacca sotto il fazzoletto o scoppia in una risata perché un vicino, appena sceso dall'autobus, è finito a gambe levate per una raffica di bora. Lei che fissa il vetro, silenziosa, impassibile, per intere sigarette, e dentro la sua faccia riflessa vede Napoli, la città mito, con la chiacchierata al Gambrinus, la sfogliatella da Scaturchio, lo struscio in via Toledo e tutto il resto della vita che non ha avuto». C'è nel gesto di nonna Lisa la stessa poetica del languore, anche nella versione gallese di hiraeth, che - mi si consenta la citazione cinematografica - sprigiona la sigaretta di Serge Reggiani nel film del regista finlandese Aki Kaurismäki, I hired a contract killer (Ho affittato un killer).

La città interiore è percorsa dall'autore ad un ritmo vertiginoso che non dà tregua, con continue accelerazioni e dietrofront e quando meno te l'aspetti, con anticipazioni che riguardano, guarda caso (ma siamo in territorio freudiano) la morte del padre («un quarantaseienne con i giorni contati»), c'è dicevo la memoria della cultura triestina, in un affresco dove appaiono personaggi indimenticabili. Eccone un campionario: Antonio Bibalo, musicista con un incredibile percorso biografico, sconosciuto in Italia ma che ha avuto la sua consacrazione in Norvegia dove è considerato l'uomo che ha rinnovato la musica scandinava. Mette in musica un racconto di Henry Miller, Il sorriso ai piedi della scala, che verrà rappresentato con grande successo, il 6 aprile 1965, allo Staatsoper di Amburgo, uno dei palcoscenici più prestigiosi del mondo. C'è l'incontro, nell'agosto del 1920, fra Umberto Saba ed Italo Svevo: «I due si imbattono per caso e si fermano a parlare, ma subito le chiacchiere precipitano in una discussione accesa. Nella versione raccolta da Quarantotti Gambini (Il poeta innamorato: ricordi), il poeta resta annichilito dall'impeto con cui lo investe lo scrittore, un accesso d'ira del tutto imprevisto. "Cossa la vol saver lei" grida Svevo, "che in afari la xe apena un pulisin! Che mi se voio posso far cussi e cussi"”. Ed ancora l'episodio che vede Quarantotti Gambini piantonare con i suoi due metri e passa d'altezza, nell'ottobre del 1941, la libreria di Umberto Saba minacciata dalle squadracce fasciste. Sempre Quarantotti Gambini, 
"antifascista antislavo, ovvero antifascista anticomunista», è responsabile di uno scontro unilatelare, visto che l'altro si limita solo ad ascoltare, con il poeta comunista Paul Eluard che appoggiava i partigiani iugoslavi. C'è poi l'emotivo pellegrinaggio alla tomba dello scrittore di origine istriana, profugo a Trieste dopo l'annessione alla Iugoslavia, Fulvio Tomizza, a Giurizzani (oggi in Croazia) nei pressi di Materada, toponimo che dà il titolo al suo primo romanzo. La tomba di Tomizza è un inno simbolico al plurilinguismo di quella zona, con le iscrizioni funebri («Passò a miglior vita», ecc.) nelle tre lingue del posto, italiano, croato e sloveno. Da citare ancora l'incontro con lo scrittore sudafricano e premio Nobel nel 2003, John Maxwell Coetzee che apre il suo saggio Lavori di scavo (Einaudi 2006) con un capitolo dedicato a Italo Svevo di cui si dichiara fervente ammiratore. Svevo ha un altro ammiratore insospettato nell'artista, pure lui sudafricano, William Kentridge (a cui si deve lo splendido esergo già citato), che è autore di una performance intitolata Confessions of Zeno, un oratorio d'ombre a base di canto, recitazione, quartetto d'archi, video e sagome di marionette che si ispira al romanzo di Svevo (i disegni sono attualmente esposti al MoMA di New York).

La scrittura vertiginosa di Covacich raggiunge il suo apice nel ricordare le innumerevoli ferite che la storia ha inflitto alla sua città. Quella, ad esempio, inflitta il 5 aprile 1945 al giovane Pino Robusti che proprio durante l'ingiusta prigionia decise, lui che non lo era, di morire da partigiano nella risiera di San Sabba dove i nazisti si macchiarono di crimini orrendi. La scia del terrore continua al di là dei confini con la morte dello scrittore e partigiano croato Ivan Goran Kovačić trucidato in Bosnia dai cetnici, i nazionalisti serbi. Accanto al suo cadavere furono trovati i fogli sparsi di un poema sorprendente, intitolato Jama, la cui prima edizione vide la luce proprio in Italia, nel 1944, con il titolo di Fossa che Covacich aggiorna in quello, ahimè, più pertinente, di Foiba. Si tratta di 63 sestine narrative che descrivono un'esecuzione in massa compiuta dagli ustascia, i fascisti croati alleati dei nazisti. "Corpi umani — racconta Covacich-cuciti l'un l'altro col fil di ferro e gettati nella fossa che hanno scavato da vivi». L'interesse dello scrittore per il poeta croato è motivato dal sospetto, infondato, di una possibile omonimia: "Una K diventata una C. All'inizio, devo ammetterlo, l'interesse per Ivan Goran Kovačić sta tutto nel viaggio tra queste due lettere, la microvariazione di un'omonimia». Così si mette un'altra volta in viaggio per cercare la tomba del poeta ma l'epoca non è la più propizia: è il 1995 e da quelle parti comincia a tirare una brutta aria, per cui meglio rinunciare e far ritorno a Trieste.

Giovanni Albertocchi Universitat de Girona 
